\title{
Employment Diversification Patterns in Pakistan: Empirical Assessment Revisited
}

\author{
Nazia Nasir ${ }^{a}$, Fouzia Yasmin $^{b}$, Noreen Safdar $^{c}$ \\ ${ }^{a}$ Research Scholar, Department of Economics, The Women University Multan, Pakistan \\ Email: Nazianasir38@gmail.com \\ ${ }^{\mathrm{b}}$ Lecturer, Department of Economics, University of Sahiwal, Pakistan \\ Email: Fouziayasmin@uosahiwal.edu.pk \\ ${ }^{c}$ Assistant Professor, Department of Economics, The Women University Multan, Pakistan \\ Email: Noreen.safdar@wum.edu.pk
}

\begin{tabular}{l}
\hline ARTICLE DETAILS \\
\hline History: \\
Accepted 15 March 2021 \\
Available Online 31 March 2021 \\
\hline Keywords: \\
Employment Diversification, \\
Seemingly Unrelated Model, Full- \\
Time, Part-Time, Sub-Sectors
\end{tabular}

JEL Classification:

Jo1, J18

DOI: $10.47067 /$ reads.v7i1.323

\begin{abstract}
Employment diversification depicts a dynamic socio-economic process where domestic workers widen the range of employment sources. Whereas, the prospect is usually based on a mix of Part-time and Fulltime employment. Employment decisions significantly derive from the economic incentives such as wage differentials and the growth rates in different sub-sectors of economic activity. Research at hand summarizes and analyses Employment Diversification Patterns in Pakistan and the motives behind the labor shift. Time series data has been collected from various sources for 1990-2018. The Seemingly unrelated regression model has been applied for empirical estimations. The current analysis of employment pattern diversification concluded that part-time and fulltime wages rates have a significant impact on the part and full-time employment in different sub-sectors of economic growth. Variation in wage rates in one sub-sector varies the employment level in different sectors. The estimates elaborated the significant rise in part-time employment these sub-sectors. Moreover, the dynamic interrelation between part-time and full-time employment is examined in the Agriculture, Construction, Electricity, Manufacturing, Wholesale and Retail Trade, Transport, Storage and Communication. These estimates show the quick adjustment of part-time employment within and across the sectors. Policies are needed to enhance labor mobility as one wants to diversify the employment one can do it to enhance the economic productivity.
\end{abstract}

(C) 2021 The authors. Published by SPCRD Global Publishing. This is an open access article under the Creative Commons Attribution-

NonCommercial 4.0

Corresponding author's email address: Fouziayasmin@uosahiwal.edu.pk

\section{Introduction}

The arrangement of employment in any economy involves many social and economic aspects. These employment patterns are expected to transfer relatively towards the industry and service away 
Review of Economics and Development Studies, Vol. 7 (1) 2021, $77-90$

from the basic agriculture economy. Previous decades illustrate some diversification of employment towards the non-agricultural sector in Pakistan (Mottaleb \& Ali, 2018). Both macro and micro-level indicators and the proportion of non-agricultural employment influence the decision of adopting a particular kind of employment (Unay-Gailhard \& Bojnec, 2019). The diversification of economic activities can occur from a process of investment and the surplus generated from agriculture and nonagriculture employment. The occupational choice consists of several inter-related decisions (Su et al., 2018). The choice to participate in economic activity may involve many activities to undertake and the nature of the economic activities, e.g. agricultural/non-agricultural, self/wage employment, FullTime/Part-Time employment (Shaheen et al., 2015). To elaborate, the basic choice of whether to participate in economic activity consists of two additional dimensions. The individual chooses the nature of the economic activity - agricultural or non-agricultural. Further, he chooses whether to specialize in one economic activity or diversify into- more than one economic activity (Khan et \& Chaudhry, 2019 \& Wang \& Sun ,2017).

When an agriculture economy diversifies, the workers may rise in status either as self-employed workers when the worker shifting from one lower-paid occupation to another high paid occupation while vice versa indicates the distress. Moreover, occupational choice and employment diversification are complex to identify (Buchenrieder and Mollers, 2006). Economic growth is highly determined by the employment level meaning that the economy is producing more valuable products and services that was not the case before the industrial revolution where the workers had no diversification choices and mainly contribute to meat, textile, and grains (Elewa \& Ezzat, 2019).

Labour allocation decisions are driven by economic incentives such as wage differentials, but also non-economic motives may play a decisive role. Research at hand summarizes theoretical insight along with presents incorporated conceptual constructions reflecting the working activities diversification, and labor shift (Chaplin et al. 2007). Möllers (2006) employment diversification is described as a dynamic socio-economic process in which domestic workers widen the range of employment sources in their portfolio. Such diversified prospect is usually based on a mix of farm and non-farm employment. Working activities diversification leads to an augmentation in the number and mix of employment sources. Thus, working activities diversification rise with the number of employment source, the equity of their allocation, and their differences. Mishra and Goodwin (1997) deal specifically with this feature that motivates domestics to become accustomed to their paid work strategies. More commonly, the suffering and demand-pull move toward allocation, with distinguishing two major inspirations of employment diversification; depends on the precise mechanism that, people may be short of diversification by adverse circumstances or pulled by the opportunity in the market of labor and high wage rate in non-farming sectors.

The labor market institutions and the policies are considered as the desired mechanism to stimulate employment generation (Westerhuis and Henrekson, 2016). Whereas the low level of employment indicates the Underutilization of the economic resources and it illustrates the space for labor absorption (Dorn \& Hanson, 2019). Furthermore, the high standard of living is associated with the stable employment level that comes from the opportunities that exist in the country and from the diversification of the employment opportunities (Nelen et al., 2013). Hence, the flexibility in the labor market results in growth led the diversification of employment pattern in the workforce (Garcia, 2016; Anxo et al.,2007). Tansel \& Acar (2017) examined the impact of labor mobility and economic globalization that is also investigated for Pakistan by (Shah \& Soomro, 2017) as well including inclusive growth. This research reconsiders the employment diversification pattern in different sub-sectors contributing to the growth of Pakistan (Anwer, 2017). In the agricultural sector modification of crops 
Review of Economics and Development Studies, Vol. 7 (1) 2021, 77-9o

from low to high value and labor-intensive crops can provide a better source of income to the farmers (Abro et al., 2010). The trade sector showed that export diversification switching from low-value-added products to high-value products increased Pakistan's growth performance (Khan et al., 2018). Similarly, the service sector contributes around 54 percent to national output as being the major employment-providing sector (Amir, 2015; Siddiqui et al., 2010).

The labor demand can generate increased geographic and industrial mobility of the labor force (Lilien, 1982; Solon, 1982) coupled with significant costs of information and mobility, these shifts can generate fluctuations in equilibrium employment that are not directly related to fluctuations in aggregate demand. Furthermore, the geographic distribution of employment may be an indication of more fundamental, permanent changes in the composition of labor demand within areas (Imran \& Arshad, 2017). Several recent studies have noted that the maintenance and continuous adaptation to diverse employment activities (Ellis, 1998, 2000; Barrett et al, 2001; Reardon et al, 2001; Lanjouw and Lanjouw, 2001; Bhaumik, 2007). Various theories are describing the behavior of employment diversification during cyclical variations (Treadway, 1969). The specific industrial employment diversification is claimed by (Buchenrieder et al. 2004).

Jouili and khemissi (2019) estimates the impact of graduated employment on the economic diversification in Saudi Arabia and come up with the conclusion that employment diversification had a positive impact on graduated students for their job creation. (Xiao et al., 2018; Batool \& Jamil, 2019) examined the drivers of industrial diversification. Dey (2018) explained that employment diversification reduces the poverty among the small and marginal landholders that further explained by Mukhtar et al., (2018) by accommodating the impact of rural-urban migration on employment quality and household welfare. Jan et al., (2012) explained the drivers of rural employment diversification in the North region of Pakistan and the Globalization was concluded as the significant factor for economic growth, provides equal opportunities to all the nation and the basis to diversify the choice of work for inclusive growth of a country. Borah (2018) elaborated on the structure of non-farm employment and identify the trend of workers moved from farm to non-farm sector and increased workforce of women due to rise in education moved them to work from primary to other sectors. The impact of education on employment diversification was studied by Reddy (2016) that showed education, employment, and expected wages affect the employment pattern in India and the similar findings are elaborated in Pakistan by (Rahman et al., 2018). Senadza (2012) had analyzed the effect of Non-farm employment diversification on the income of rural people of Ghana and come up with that wage employment and non-farm employment positively affect the income. Many empirical studies elaborated the employment diversification pattern from the Primary Sector to the non-farm secondary sector and lead to the growth-led diversification. Therefore, the employment diversification depicts a dynamic socio-economic practice where native workers are provided with the diverse range of employment opportunities. Employment decisions significantly derive from the economic incentives such as wage differentials and the growth rates in different sub-sectors of economic activity. So, the analysis ahead will focus on the prospects of labor shift as a mix of Part-time and Full-time employment and employment Diversification Patterns in Pakistan.

\section{Data and Methodology}

Research at hand used annual time series data of 1990-2018 for employment rate, real wage rate, GDP growth rate, and labor participation rate of six sub-sectors e.g. agriculture, construction, manufacturing, trade, and wholesale, electricity and gas distribution, and Transport, storage and communication. The analysis has been done by using the most appropriate approaches OLS and SUR (Seemingly unrelated regression) model that also bridges the gap of the previous methodologies used in 
literature. The current analysis of the employment diversification pattern in Pakistan used the Sectoral employment (full-time and part-time employment) as the regressand that is followed by the list of control variables e.g. wage rate and Sectoral growth.

\section{Model specification}

\subsection{Theoretical Model specification}

This section incorporates the key details of the theoretical and the empirical model specification. SUR (seemingly unrelated regression) or SURE (seemingly unrelated regression equation) proposed by (Zellner, 1962) consists of various linear regression equations having their dependent variable and different sets of explanatory variables. The linear regression model for $k$ observation can be expressed as:

$$
Y_{k l}=x_{k l}^{\prime} \gamma_{l}+\varepsilon_{k l}
$$

In matrix form, the SUR model is expressed as:

$$
\left[\begin{array}{c}
Y_{1} \\
Y_{2} \\
\vdots \\
Y_{n}
\end{array}\right]=\left[\begin{array}{cccc}
X_{1} & 0 & 0 & 0 \\
0 & x_{2} & 0 & 0 \\
0 & 0 & \ddots & 0 \\
0 & 0 & 0 & X_{n}
\end{array}\right]\left[\begin{array}{c}
\gamma_{1} \\
\gamma_{2} \\
\vdots \\
\gamma_{n}
\end{array}\right]+\left[\begin{array}{c}
\mathcal{E}_{1} \\
\mathcal{E}_{2} \\
\vdots \\
\mathcal{E}_{n}
\end{array}\right]
$$

The above model shows that the error term has a zero mean and homoscedastic relation among the variables. But across equations, it correlates as follows:

$$
E\left(\varepsilon_{k l} \varepsilon_{k l} \mid X\right)=\sigma_{l l^{\prime}} \text { and } \sigma_{l l^{\prime}} \neq 0 \text { where } l \neq l^{\prime}
$$

In this scenario, the error term $\mathcal{E}_{l}$ assumes the following conditions:

i. $\quad$ Mean of $\varepsilon_{l:} E\left(\varepsilon_{l} \mid X\right)=0$

ii. $\quad$ Variance of $\mathcal{E}_{l}: E\left(\mathcal{E}_{l} \varepsilon_{l}^{\prime} \mid \mathcal{X}\right)=\sigma_{l l} I_{M}$

iii. Co-variance of $\mathcal{E}_{l}$ across equations $l$ and $l^{\prime}: \quad E\left(\varepsilon_{l} \varepsilon_{l^{\prime}}^{\prime} \mid X\right)=\sigma_{l l^{\prime}} I_{M}$ where $l \neq l^{\prime}$

iv. Overall variance-covariance matrix: $\Omega=E(\mathcal{E} \mathcal{E})=\Sigma \otimes I_{M}$

However, the estimation of the SUR model consists of two stages: In the first stage, each equation of the system is estimated by regressing using OLS. This obtains the estimator $\hat{\gamma}_{l}$ with a separate residual term. These terms are used to compute $\Sigma$ which operates in the next stage:

$$
\begin{gathered}
\hat{\varepsilon}_{l}=Y_{l}-x_{l} \widehat{\gamma}_{l} \\
\hat{\gamma}_{l}=\left(X^{\prime} X\right)^{-1} X^{\prime} Y \text { and } \hat{\sigma}_{l l^{\prime}}=\hat{\varepsilon}_{l}^{\prime} \hat{\varepsilon}_{l^{\prime}} / M
\end{gathered}
$$

In the second stage, the computed value of $\hat{\Sigma}$ is substituted into $\Sigma$ of the GLS estimator $\hat{\gamma}$, which considers an optimal estimator from that estimator, yield by OLS consistent estimators of each equation. The GLS estimator and its variance can be expressed as:

$$
\begin{gathered}
\left.\hat{\gamma}=\left\{X^{\prime}\left(\Sigma^{-1} \otimes I_{M}\right) X\right\}^{-1}\left\{X^{\prime}\left(\Sigma^{-1} \otimes I_{M}\right) Y\right)\right\} \\
V(\hat{\gamma})=\left\{X^{\prime}\left(\Sigma^{-1} \otimes I_{M}\right) X\right\}^{-1}
\end{gathered}
$$

By putting values, we get

$$
\left.\hat{\gamma}=\left\{X^{\prime}\left(\hat{\Sigma}^{-1} \otimes I_{M}\right) X\right\}^{-1}\left\{X^{\prime}\left(\hat{\Sigma}^{-1} \otimes I_{M}\right) Y\right)\right\}
$$


Review of Economics and Development Studies, Vol. 7 (1) 2021, 77-90

From the above GLS estimator, we acquire a SUR estimator for the model, which is given below:

$$
\hat{\gamma}_{S U R}=\left\{X^{\prime}\left(\hat{\Sigma} \otimes I_{M}\right)^{-1} X\right\}^{-1}\left\{X^{\prime}\left(\hat{\Sigma} \otimes I_{M}\right)^{-1} Y\right\}
$$

The SUR model proceeds when linear equations are correlated only through their error terms. Its parametric estimate differs from one equation to another, but the fluctuation in repressors depends on the nature of the model.

\subsection{Empirical Model Specification}

The empirical model of employment pattern diversification incorporates the following details: wage rate and share of GDP of each sub-sector are the control variables and employment (the dependent variable of each sub-sector) has two-dimension i.e. part-time and full-time employment. This model used the two time-lagged wage rates and one lagged employment rate. Full-time employment is denoted by $e m_{F t}$ and part-time employment by $e m_{P t}$ where the parttime and full-time wages, the share of GDP are donated by $w_{p t}, w_{f t}, \emptyset G D P$ respectively.

Model 1: Employment in the Agriculture sector

Agri em $\mathrm{Ft}_{\mathrm{t}}=A g \alpha_{a a} e m_{F t-1}+A g \alpha_{a b} e m_{P t-1}+A g \beta_{1} w_{F t-1}+A g \beta_{2} w_{F t-2}+A g \varphi G D P+\epsilon$

Agri em $\mathrm{Pt}_{\mathrm{t}}=A g \alpha_{b a} e m_{P t-1}+A g \alpha_{b b} e m_{F t-1}+A g \gamma_{1} w_{P t-1}+A g \gamma_{2} w_{P t-2}+A g \varphi G D P+\epsilon$

Model 2: Employment in the Manufacturing sector

$M n e m_{F t}=M n \alpha_{a a} e m_{F t-1}+M n \alpha_{a b} e m_{P t-1}+M n \beta_{1} w_{F T-1}+M n \beta_{2} w_{F t-2}+M n \varphi G D P+\mu$

$M n e m_{P t}=M n \alpha_{b a} e m_{P t-1}+M n \alpha_{b b} e m_{F t-1}+M n \gamma_{1} w_{P t-1}+M n \gamma_{2} w_{P t-2}+M n \varphi G D P+\mu$

Model 3: Employment in Construction Sector

Cn em $m_{F t}=C n \alpha_{a a} e m_{F t-1}+C n \alpha_{a b} e m_{P t-1}+C n \beta_{1} w_{F t-1}+C n \beta_{2} w_{F t-2}+C n \varphi G D P+\sigma$

$C n e m_{P t}=C n \alpha_{b a} e m_{P t-1}+C n \alpha_{b b} e m_{F t-1}+C n \gamma_{1} w_{P t-1}+C n \gamma_{2} w_{P t-2}+C n \varphi G D P+\sigma$

Model 4: Employment in Wholesale and Retail Trade Sector

$W R e m_{F t}=W R \alpha_{a a} e m_{F t-1}+W R \alpha_{a b} e m_{P t-1}+W R \beta_{1} w_{F t-1}+W R \beta_{2} w_{F t-2}+W R \varphi G D P+\rho$ (7)

$W R e m_{P t}=W R \alpha_{b a} e m_{P t-1}+W R \alpha_{b b} e m_{F t-1}+W R \gamma_{1} w_{P t-1}+W R \gamma_{2} w_{P t-2}+W R \varphi G D P+\rho$

Model 5: Employment in Electricity and Gas Distribution Sector

$E G e m_{F t}=E G \alpha_{a a} e m_{F t-1}+E G \alpha_{a b} e m_{P t-1}+E G \beta_{1} w_{F t-1}+E G \beta_{2} w_{F t-2}+E g \varphi G D P+\vartheta$

$E G e m_{P t}=E G \alpha_{b a} e m_{P t-1}+E G \alpha_{b b} e m_{F t-1}+E G \gamma_{1} w_{P t-1}+E G \gamma_{2} w_{P t-2}+E g \varphi G D P+\vartheta$

Model 6: Employment in Transport, storage and communication Sector

Tran $e m_{F t}=\operatorname{Tr} \alpha_{a a} e m_{F t-1}+\operatorname{Tr} \alpha_{a b} e m_{P t-1}+\operatorname{Tr} \beta_{1} w_{F t-1}+\operatorname{Tr} \beta_{2} w_{F t-2}+\operatorname{Tr} \varphi G D P+\tau$

Tran $e m_{P t}=\operatorname{Tr} \alpha_{a b} e m_{P t-1}+\operatorname{Tr} \alpha_{b b} e m_{F t-1}+\operatorname{Tr} \gamma_{1} w_{P t-1}+\operatorname{Tr} \gamma_{2} w_{P t-2}+\operatorname{Tr} \varphi G D P+\tau$ 
Table 1: Descriptive Analysis of the data by Sectors

\begin{tabular}{|c|c|c|c|c|c|c|c|c|c|c|c|}
\hline \multicolumn{2}{|c|}{ Agriculture } & \multicolumn{2}{|c|}{$\begin{array}{c}\text { Electricity and Gas } \\
\text { Distribution }\end{array}$} & \multicolumn{2}{|c|}{ Manufacturing } & \multicolumn{2}{|c|}{ Construction } & \multicolumn{2}{|c|}{$\begin{array}{c}\text { Wholesale and } \\
\text { Retail Trade }\end{array}$} & \multicolumn{2}{|c|}{$\begin{array}{l}\text { Transport, storage } \\
\text { and communication }\end{array}$} \\
\hline Mean & Std. Dev. & Mean & Std.Dev. & Mean & Std.Dev & Mean & Std.Dev & Mean & Std.Dev & Mean & Std.Dev \\
\hline $3 \cdot 547$ & 3.660 & 2.829 & 0.918 & 15.686 & 2.121 & 2.859 & 0.767 & $17 \cdot 353$ & 1.100 & 11.145 & 1.397 \\
\hline 22.907 & 2.294 & $4 \cdot 305$ & 20.141 & $5 \cdot 300$ & 3.980 & 3.632 & 8.311 & 4.070 & 2.859 & 4.111 & 1.977 \\
\hline 807.083 & 606.644 & $7 \cdot 45^{2}$ & 6.128 & 180.488 & 160.243 & 54.428 & 49.044 & 47.246 & 40.366 & 1.011 & 0.695 \\
\hline 4.912 & 0.309 & 0.053 & 0.103 & $\begin{array}{c}79454.35 \\
\text { o }\end{array}$ & $\begin{array}{c}54916.39 \\
\text { o }\end{array}$ & $\begin{array}{c}69892.40 \\
o\end{array}$ & $\begin{array}{c}53655.51 \\
\text { o }\end{array}$ & $\begin{array}{c}65883.62 \\
o\end{array}$ & $\begin{array}{c}44196.16 \\
\text { o }\end{array}$ & $\begin{array}{c}93465.06 \\
\text { o }\end{array}$ & $\begin{array}{c}63858.43 \\
0\end{array}$ \\
\hline $\begin{array}{c}46083.10 \\
0\end{array}$ & $\begin{array}{c}29723.10 \\
0\end{array}$ & $\begin{array}{c}147658.90 \\
0\end{array}$ & $\begin{array}{c}117967.20 \\
0\end{array}$ & 0.579 & 0.113 & 0.349 & 0.075 & 0.322 & 0.133 & 0.090 & 0.039 \\
\hline 9.267 & 1.147 & 0.416 & 0.120 & 4.828 & 0.339 & 3.282 & 0.389 & 2.979 & 0.254 & 1.197 & 0.472 \\
\hline
\end{tabular}

Source: Estimation by the author using STATA 14.1 
Review of Economics and Development Studies, Vol. 7 (1) 2021, 77-90

Table 2: Correlation of Employment by Sectors (Full Time and Part-Time)

\begin{tabular}{|c|c|c|c|c|c|}
\hline \multicolumn{6}{|c|}{ Agriculture } \\
\hline & AGR & ASH & FTAE & PTAE & FTAW \\
\hline ASH & 0.233 & & & & \\
\hline FTAE & 0.093 & 0.752 & & & \\
\hline PTAE & 0.185 & 0.180 & -0.032 & & \\
\hline FTAW & -0.237 & -0.831 & -0.494 & -0.287 & \\
\hline PTAW & -0.237 & -0.810 & -0.594 & -0.281 & 0.906 \\
\hline \multicolumn{6}{|c|}{ Construction } \\
\hline & CGR & $\mathrm{CSH}$ & FTCE & PTCE & FTCW \\
\hline $\mathrm{CSH}$ & -0.014 & & & & \\
\hline FTCE & 0.022 & 0.203 & & & \\
\hline PTCE & 0.236 & 0.205 & -0.344 & & \\
\hline FTCW & 0.031 & -0.317 & 0.717 & -0.454 & \\
\hline PTCW & -0.017 & -0.403 & 0.604 & -0.435 & 0.972 \\
\hline \multicolumn{6}{|c|}{ Electricity and gas } \\
\hline & EGR & ESH & FTELE & PTELE & FTELW \\
\hline ESH & 0.246 & & & & \\
\hline FTELE & 0.129 & 0.644 & & & \\
\hline PTELE & -0.050 & -0.188 & -0.468 & & \\
\hline FTELW & 0.027 & -0.795 & -0.663 & 0.230 & \\
\hline PTELW & 0.037 & -0.793 & -0.658 & 0.240 & 0.997 \\
\hline \multicolumn{6}{|c|}{ Manufacturing } \\
\hline & MGR & MSH & FTME & PTME & FTMW \\
\hline MSH & 0.466 & & & & \\
\hline FTME & 0.281 & -0.255 & & & \\
\hline PTME & 0.225 & -0.112 & 0.720 & & \\
\hline FTMW & -0.364 & -0.760 & 0.345 & 0.082 & \\
\hline PTMW & -0.335 & -0.771 & 0.362 & 0.124 & 0.975 \\
\hline \multicolumn{6}{|c|}{ Transport } \\
\hline & TGR & TSH & FTTE & PTTE & FTTW \\
\hline TSH & -0.029 & & & & \\
\hline FTTE & 0.358 & -0.337 & & & \\
\hline PTTE & 0.368 & -0.384 & 0.581 & & \\
\hline FTTW & -0.175 & 0.794 & -0.560 & -0.654 & \\
\hline PTTW & -0.173 & 0.652 & -0.071 & -0.315 & 0.722 \\
\hline \multicolumn{6}{|c|}{ Wholesale and Retail Trade } \\
\hline & WGR & WSH & FTWE & PTWE & FTWW \\
\hline WSH & 0.359 & & & & \\
\hline FTWE & -0.051 & 0.008 & & & \\
\hline PTWE & 0.055 & -0.478 & -0.106 & & \\
\hline FTWW & 0.026 & 0.606 & 0.257 & -0.638 & \\
\hline PTWW & -0.033 & 0.429 & 0.329 & -0.577 & 0.844 \\
\hline
\end{tabular}

Source: Estimation by the author using STATA 14.1 


\section{Empirical Analysis}

Table 3 shows that the first lag of wage has a negative and significant impact on full-time employment in the agricultural sector. As it is observed that Wages play the most significant role in determining employment. If one is paid more he will be more motivated towards work and it enhances the productivity level (Jan et al., 2012). The second lag of wages has a positive and significant impact on full-time employment in the agricultural sector with the probability of 0.0001 units. As most of the studies reveal that if the wages rise the workers who are sitting idle at home will prefer to do jobs (Qureshi \& Ghani, 1989; Card \& Krueger, 1992). Part-time wages both the included lags have not a significant impact on agricultural employment due to the limited resources of rural areas. It is observed that most of the people especially women participation in rural employment gives them too much low reward in the form of pay (Jan et al., 2012). Full-time and part-time agricultural employment changes when wages changes (Ghilarducci, 2018). Lack of infrastructure, modern technology, and low level of education are the main factors for the low productivity and employment diversification (Batool \& Jamil, 2019). 
Table 3: Empirical Estimates using SUR of Full Time Employment Diversification

\begin{tabular}{|c|c|c|c|c|c|c|}
\hline Variables & Agriculture & Construction & Electricity & $\begin{array}{c}\text { Manufacturi } \\
\text { ng }\end{array}$ & $\begin{array}{c}\text { Wholesale And } \\
\text { Retail Trade }\end{array}$ & $\begin{array}{c}\text { Transport, } \\
\text { Storage, And } \\
\text { Communicatio } \\
\text { n }\end{array}$ \\
\hline & $\begin{array}{l}\text { Coefficients } \\
\text { (P-Value) }\end{array}$ & $\begin{array}{l}\text { Coefficients } \\
\text { (P-Value) }\end{array}$ & $\begin{array}{c}\text { Coefficients } \\
\text { (P-Value) }\end{array}$ & $\begin{array}{l}\text { Coefficients } \\
\text { (P-Value) }\end{array}$ & $\begin{array}{l}\text { Coefficients } \\
\text { (P-Value) }\end{array}$ & $\begin{array}{l}\text { Coefficients } \\
\text { (P-Value) }\end{array}$ \\
\hline Full Time Wage (-1) & $\begin{array}{l}-39.336 \\
(0.008)\end{array}$ & $\begin{array}{c}0.308 \\
(0.073)\end{array}$ & $\begin{array}{c}0.002 \\
(0.009)\end{array}$ & $\begin{array}{c}0.532 \\
(0.025)\end{array}$ & $\begin{array}{c}0.722 \\
(0.005)\end{array}$ & $\begin{array}{c}0.001 \\
(0.013)\end{array}$ \\
\hline Full Time Wage (-2) & $\begin{array}{l}40.493 \\
(0.000)\end{array}$ & $\begin{array}{c}-0.217 \\
(0.006)\end{array}$ & $\begin{array}{l}-4.2137 \\
(0.018)\end{array}$ & $\begin{array}{c}1.16 \mathrm{E}-\mathrm{o} 6 \\
(0.736)\end{array}$ & $\begin{array}{c}-1.165 \\
(0.000)\end{array}$ & $\begin{array}{l}-0.000 \\
(0.029)\end{array}$ \\
\hline Part Time Wage (-1) & $\begin{array}{c}2.12 \mathrm{E}-05 \\
(0.970)\end{array}$ & $\begin{array}{l}-0.003 \\
(0.566)\end{array}$ & $\begin{array}{l}12.064 \\
(0.001)\end{array}$ & $\begin{array}{c}1.76 \mathrm{E}-\mathrm{o} 6 \\
(0.488)\end{array}$ & $\begin{array}{c}0.755 \\
(0.029)\end{array}$ & $\begin{array}{c}0.001 \\
(0.037)\end{array}$ \\
\hline Part Time Wage (-2) & $\begin{array}{l}-0.000 \\
(0.722)\end{array}$ & $\begin{array}{c}0.003 \\
(0.551)\end{array}$ & $\begin{array}{l}-8.255 \\
(0.000)\end{array}$ & $\begin{array}{l}-0.967 \\
(0.000)\end{array}$ & $\begin{array}{l}-1.303 \\
(0.001)\end{array}$ & $\begin{array}{c}-0.001 \\
(0.020)\end{array}$ \\
\hline $\begin{array}{l}\text { Full Time } \\
\text { Employment (-1) }\end{array}$ & $\begin{array}{c}0.742 \\
(0.000)\end{array}$ & $\begin{array}{c}0.503 \\
(0.053)\end{array}$ & $\begin{array}{c}0.000 \\
(0.267)\end{array}$ & $\begin{array}{c}0.001 \\
(0.007)\end{array}$ & $\begin{array}{c}0.000 \\
(0.895) \\
\end{array}$ & $\begin{array}{c}0.000 \\
(0.183)\end{array}$ \\
\hline $\begin{array}{l}\text { Part Time } \\
\text { Employment (-1) }\end{array}$ & $\begin{array}{c}0.132 \\
(0.773) \\
\end{array}$ & $\begin{array}{l}-0.302 \\
(0.703)\end{array}$ & $\begin{array}{c}0.001 \\
(0.017) \\
\end{array}$ & $\begin{array}{c}-0.001 \\
(0.000)\end{array}$ & $\begin{array}{l}-0.225 \\
(0.728)\end{array}$ & $\begin{array}{c}5.786 \\
(0.054) \\
\end{array}$ \\
\hline Growth Rate & $\begin{array}{c}-0.428 \\
(0.004) \\
\end{array}$ & $\begin{array}{c}0.002 \\
(0.754) \\
\end{array}$ & $\begin{array}{l}-0.003 \\
(0.005) \\
\end{array}$ & $\begin{array}{c}0.527 \\
(0.027) \\
\end{array}$ & $\begin{array}{l}-0.000 \\
(0.972) \\
\end{array}$ & $\begin{array}{c}0.008 \\
(0.877) \\
\end{array}$ \\
\hline C & $\begin{array}{c}1.683 \\
(0.624) \\
\end{array}$ & $\begin{array}{c}1.531 \\
(0.059) \\
\end{array}$ & $\begin{array}{c}0.221 \\
(0.077)\end{array}$ & $\begin{array}{c}-0.000 \\
(0.884)\end{array}$ & $\begin{array}{c}1.746 \\
(0.008)\end{array}$ & $\begin{array}{c}0.225 \\
(0.619)\end{array}$ \\
\hline
\end{tabular}

Source: Estimation by the author using STATA 14.1. 
Table 4: Empirical Estimates using SUR of Part-Time employment diversification

\begin{tabular}{|c|c|c|c|c|c|c|}
\hline Variables & Agriculture & Construction & Electricity & Manufacturing & $\begin{array}{c}\text { Wholesale And } \\
\text { Retail Trade }\end{array}$ & $\begin{array}{c}\text { Transport, } \\
\text { Storage, And } \\
\text { Communication }\end{array}$ \\
\hline & $\begin{array}{l}\text { Coefficients } \\
\text { (P-Value) }\end{array}$ & $\begin{array}{l}\text { Coefficients } \\
\text { (P-Value) }\end{array}$ & $\begin{array}{l}\text { Coefficients } \\
\text { (P-Value) }\end{array}$ & $\begin{array}{l}\text { Coefficients } \\
\text { (P-Value) }\end{array}$ & $\begin{array}{l}\text { Coefficients } \\
\text { (P-Value) }\end{array}$ & $\begin{array}{l}\text { Coefficients } \\
\text { (P-Value) }\end{array}$ \\
\hline Full Time Wage (-1) & $\begin{array}{c}0.308 \\
(0.073)\end{array}$ & $\begin{array}{c}-5.19 \mathrm{E}-07 \\
(0.055)\end{array}$ & $\begin{array}{c}-5.19 \mathrm{E}-\mathrm{O} 7 \\
(0.055)\end{array}$ & $\begin{array}{c}0.107 \\
(0.246) \\
\end{array}$ & $\begin{array}{c}0.203 \\
(0.053)\end{array}$ & $\begin{array}{c}0.002 \\
(0.074)\end{array}$ \\
\hline Full Time Wage (-2) & $\begin{array}{c}-0.217 \\
(0.006)\end{array}$ & $\begin{array}{c}5.459 \\
(0.000)\end{array}$ & $\begin{array}{c}5.459 \\
(0.000)\end{array}$ & $\begin{array}{c}-5 \cdot 32 \mathrm{E}-06 \\
(0.045)\end{array}$ & $\begin{array}{c}-7.44 \mathrm{E}-07 \\
(0.798)\end{array}$ & $\begin{array}{l}-0.002 \\
(0.022)\end{array}$ \\
\hline Part Time Wage (-1) & $\begin{array}{c}0.603 \\
(0.087)\end{array}$ & $\begin{array}{l}-0.000 \\
(0.857) \\
\end{array}$ & $\begin{array}{l}-0.000 \\
(0.857) \\
\end{array}$ & $\begin{array}{c}1.59 \mathrm{E}-\mathrm{o} 6 \\
(0.475) \\
\end{array}$ & $\begin{array}{c}-7.89 \mathrm{E}-\mathrm{O} 7 \\
(0.817) \\
\end{array}$ & $\begin{array}{c}-4.732 \\
(0.028) \\
\end{array}$ \\
\hline Part Time Wage (-2) & $\begin{array}{l}-0.341 \\
(0.077)\end{array}$ & $\begin{array}{l}-0.000 \\
(0.566) \\
\end{array}$ & $\begin{array}{l}-0.000 \\
(0.566) \\
\end{array}$ & $\begin{array}{c}0.001 \\
(0.095) \\
\end{array}$ & $\begin{array}{l}-14.054 \\
(0.183) \\
\end{array}$ & $\begin{array}{c}8.832 \\
(0.013) \\
\end{array}$ \\
\hline Full Time Employment (-1) & $\begin{array}{l}-0.306 \\
(0.096)\end{array}$ & $\begin{array}{c}0.115 \\
(0.068) \\
\end{array}$ & $\begin{array}{c}0.115 \\
(0.068) \\
\end{array}$ & $\begin{array}{c}0.764 \\
(0.000)\end{array}$ & $\begin{array}{l}-0.662 \\
(0.081)\end{array}$ & $\begin{array}{l}-7.219 \\
(0.019)\end{array}$ \\
\hline Part Time Employment (-1) & $\begin{array}{c}0.315 \\
(0.080) \\
\end{array}$ & $\begin{array}{c}27.194 \\
(0.000) \\
\end{array}$ & $\begin{array}{c}27.194 \\
(0.000) \\
\end{array}$ & $\begin{array}{l}-0.879 \\
(0.000)\end{array}$ & $\begin{array}{c}0.304 \\
(0.244) \\
\end{array}$ & $\begin{array}{c}4.820 \\
(0.034)\end{array}$ \\
\hline Growth Rate & $\begin{array}{c}0.009 \\
(0.640) \\
\end{array}$ & $\begin{array}{c}0.001 \\
(0.250) \\
\end{array}$ & $\begin{array}{c}0.001 \\
(0.250) \\
\end{array}$ & $\begin{array}{l}-0.000 \\
(0.861) \\
\end{array}$ & $\begin{array}{c}-9.53 \mathrm{E}-05 \\
(0.988) \\
\end{array}$ & $\begin{array}{c}-2.727 \\
(0.085) \\
\end{array}$ \\
\hline C & $\begin{array}{c}3.871747 \\
(0.035)\end{array}$ & $\begin{array}{c}0.505 \\
(0.011)\end{array}$ & $\begin{array}{c}0.505 \\
(0.011)\end{array}$ & $\begin{array}{l}-0.006 \\
(0.985)\end{array}$ & $\begin{array}{l}-0.244 \\
(0.309)\end{array}$ & $\begin{array}{l}-0.005 \\
(0.974)\end{array}$ \\
\hline
\end{tabular}

Source: Estimation by the author using STATA 14.1 
Review of Economics and Development Studies, Vol. 7 (1) 2021, 77-90

The first lag wages have a positive and significant impact on full-time employment in the construction sector. The construction sector and construction activities are considered as one of the major sources of income (Qureshi \& Ghani, 1989 ; Ghilarducci, 2018 ; Philips, 1958). Part-time one and two time-lagged wages have a significant impact on construction employment because people prefer part-time jobs due to the high wage rate per hour which results in decline in parttime labor demand (Houseman \& Osawa, 1995). Construction plays an important role in uplift economic development and growth it is regarded as a mechanism to generate employment opportunities for millions of unskilled, semi-skilled, and skilled labor forces. Therefore, the impact of construction employment on growth is not significant due to economic and political disturbance. In the electricity and gas distribution sector the wages have a positive and significant impact on previous full and part-time electricity employment. Electricity reform programs included privatization of state-owned enterprises played a very important role in economic development. The labor force working in electricity sectors especially in private companies get more employment benefit. Therefore, electricity sectors play a vital role in economic development (Qudrat-Ullah, 2015 ; Joskow, 2006). One lag wages have a positive and statistically significant impact on part-time and full-time employment in the manufacturing sector. Manufacturing is the third-largest sector of Pakistan most of the labor force got employment in this sector. One time lag wages have not significant impact while the second lag of wages has a significant impact on employment in the manufacturing sector. On the other hand, part-time second lag of wages has a positive and statically significant impact on employment (Sheikh et al.,1992). The lag wages have a positive and significant impact on full-time and parttime employment in the wholesale and retail sector. Two-time period lagged wages have not significant of the part-time employees in the wholesale and retail sector as found by (Ahmed et al., 2012). The transport, storage, and communication sector of Pakistan play an important role in economic development and it is also important for improving the competitiveness of the country's export. Full-time and part-time transport sector employment has a positive and significant impact. Both the one and two-time lag wages of Full time and part-time have also a positive impact on employment. Sustainable economic development is dependent on the low-cost transport and logistic sector. Modernizing the transport sector through the continuous process of reform supported by focused investment in all of its sub-sectors. But due to political instability and disturbance in internal economic affairs transport sector becomes stagnant toward economic growth (Ahmed \& Ahsan, 2011).

\section{Conclusion and Policy Recommendation}

The current analysis of employment pattern diversification concluded that part-time and full-time wages rates have a significant impact on the part and full-time employment in different sub-sectors of economic growth. Variation in wage rates in one sub-sector varies the employment level in different sectors. Workers switch from one employment sector to another sector. Parttime employment switching reduces the cost involved as well for employer's searches for seasonal demand of labor. It forecasts the quick adjustment for part-time labor instead of full-time labor, which notices fewer wages and discards from social assistance. This low-income labor helps sectors to maximize their profits as well as minimize their production costs. The estimates are relative to overall employment elaborate the significant rise in part-time work like construction, wholesale and retail trade, transport sectors. These estimates show the quick adjustment of parttime employment within and across the sectors. Moreover, the dynamic interrelation between part-time and full-time employment is examined in the construction, manufacturing wholesale and retail trade, and transport divisions. Based on these estimates, the outcome is that part-time employment acts as a challenging variable concerning full-time employment, due to its availability 
Review of Economics and Development Studies, Vol. 7 (1) 2021, 77-90

on simple and manageable conditions in sectors. Employment needs to enhance the percentage of employment ratio with the growth ratio of the workforce. Policies are needed to lower the cost associated with the employment switch. Policies are needed to enhance labor mobility as one wants to diversify the employment one can do it to enhance the economic productivity. The government may revise the policies for part-time workers by introducing the minimum wage based on work hours that may protect the rights of the workforce as per their production activity.

\section{References}

Abro, A., \& Sadaqat, M. (2010). Poverty Alleviation through diversifying towards high value crops in Pakistan. Int. J. Buss. Mgt. Eco. Res, 1(1), 1-8.

Ahmed, A. M., Andersson, L., \& Hammarstedt, M. (2012). Does age matter for employability? A field experiment on ageism in the Swedish labour market. Applied Economics Letters, 19(4), 403-406.

Ahmed, A., \& Ahsan, H. (2011). Contribution of services sector in the economy of Pakistan. Working Papers \& Research Reports, 2011.

Amir, A. F., Abd Ghapar, A., Jamal, S. A., \& Ahmad, K. N. (2015). Sustainable tourism development: A study on community resilience for rural tourism in Malaysia. Procedia-Social and Behavioral Sciences, 168, 116-122.

Anwar, Y. (2017). Does Labor Mobility and Economic Globalization Matter for Inclusive Growth? Evidence from Pakistan. Stud, 6(3).

Anxo, D., Fagan, C., Cebrian, I., \& Moreno, G. (2007). Patterns of labour market integration in Europe-a life course perspective on time policies. Socio-Economic Review, 5(2), 233-26o.

Anxo, D., Flood, L., Mencarini, L., Pailhé, A., Solaz, A., \& Tanturri, M. L. (2007). Time allocation between work and family over the life-cycle: A comparative gender analysis of Italy, France, Sweden and the United States.

Anxo, D., Hussain, S., \& Shukur, G. (2012). The demand of part-time in European companies: a multilevel modelling approach. Applied Economics, 44(8), 1057-1066.

Arif, B. W. (2012). The nexus between labor reallocation and sectoral productivities in Pakistan: 1980 to 2007. International Journal of Peace and Development Studies, 3(5), 85-91.

Barrett, C. B., Reardon, T., \& Webb, P. (2001). Nonfarm income diversification and household livelihood strategies in rural Africa: concepts, dynamics, and policy implications. Food policy, 26(4), 315-331.

Batool, S., \& Jamil, F. (2019). Rural employment and income diversification in Pakistan. Pakistan Journal of Agricultural Sciences, 56(2).

Bhaumik, S. K. (2007). Diversification of employment and earnings by rural households in West Bengal. Indian Journal of Agricultural Economics, 62(4), 585.

Borah, N., Rabha, D., \& Athokpam, F. D. (2016). Tree species diversity in tropical forests of Barak valley in Assam, India. Tropical Plant Research, 3(1), 1-9.

Buchenrieder, G., \& Mollers, J. (2006). A synthesis of theoretical concepts for analysing non-farm rural employment (No. 1004-2016-78607).

Buchenrieder, G., Knerr, B., \& Kirk, M. (2004). Poverty impacts and policy options of non-farm rural employment. Margraf Publishers.

Card, D., \& Krueger, A. B. (1992). School quality and black-white relative earnings: A direct assessment. The quarterly journal of Economics, 107(1), 151-200.

Chaplin, H., Gorton, M., \& Davidova, S. (2007). Impediments to the diversification of rural economies in central and eastern europe: evidence from small-scale farms in Poland. Regional Studies, 41(3), 361-376.

Dorn, D., \& Hanson, G. (2019). When work disappears: Manufacturing decline and the falling 
Review of Economics and Development Studies, Vol. 7 (1) 2021, 77-90

marriage market value of young men. American Economic Review: Insights, 1(2), 161-78.

Elewa, A., \& Ezzat, R. A. (2019). Trade liberalization, domestic competition, and total factor productivity: Evidence from manufacturing sector. EconWorld2019.

Ellis, F. (1998). Household strategies and rural livelihood diversification. The journal of development studies, 35(1), 1-38.

Ellis, F. (2000). The determinants of rural livelihood diversification in developing countries. Journal of agricultural economics, 51(2), 289-302.

Fritzsch, J., Möllers, J., \& Buchenrieder, G. (2011). Employment diversification of farm households and structural change in the rural economy of the New Member States (No. 134). Discussion Paper, Leibniz Institute of Agricultural Development in Central and Eastern Europe.

García-López, M. Á., \& Moreno-Monroy, A. I. (2016). Income segregation and urban spatial structure: evidence from Brazil.

Ghilarducci, T., \& Webb, A. (2018). The distribution of time in retirement: Evidence from the health and retirement survey. Work, Aging and Retirement, 4(3), 251-261.

Goodwin, B. K., \& Mishra, A. K. (2006). Are “decoupled" farm program payments really decoupled? An empirical evaluation. American Journal of Agricultural Economics, 88(1), 7389.

Houseman, S., \& Osawa, M. (1995). Part-time and temporary employment in Japan. Monthly Lab. Rev., 118, 10.

Imran, S., \& Arshad, R. M. I. (2017). Impact of Microfinance on Income and Employment in Pakistan: A Primary Data Analysis. Review of Economics and Development Studies, 3(1), 69-82.

Jan, I., Khattak, M. K., Khan, M. A., Hayat, S., \& Rahim, T. (2012). Factors affecting rural livelihood choices in Northwest Pakistan. Sarhad Journal of Agriculture, 28(4), 681-688.

Joskow, P. L. (2006). Competitive electricity markets and investment in new generating capacity. AEI-Brookings Joint Center Working Paper, (o6-14).

Joskow, P., \& Tirole, J. (2006). Retail electricity competition. The RAND Journal of Economics, 37(4), 799-815.

Jouili, T. A., \& Khemissi, F. M. (2019). Impact of economic diversification on graduates employment.

Jouili, T. A., \& Khemissi, F. M. (2019). Impact of economic diversification on graduates employment.

Khan, A. Q., Hafeez, M. H., Saleem, N., \& Azam, M. (2018). Exploring the impact of financial development on inequality: evidence from three Asian countries. Review of Economics and Development Studies, 4(2), 341-355.

Khan, R., \& Chaudhry, I. S. (2019). Impact of human capital on employment and economic growth in developing countries. Review of Economics and Development Studies, 5(3), 487-496.

Künn-Nelen, A., De Grip, A., \& Fouarge, D. (2013). Is part-time employment beneficial for firm productivity?. ILR Review, 66(5), 1172-1191.

Lanjouw, J. O., \& Lanjouw, P. (2001). The rural non-farm sector: issues and evidence from developing countries. Agricultural economics, 26(1), 1-23.

Lilien, D. M. (1982). Sectoral shifts and cyclical unemployment. Journal of political economy, 90(4), 777-793.

Mottaleb, K. A., \& Ali, A. (2018). Rural livelihood diversification strategies and household welfare in Bhutan. The European Journal of Development Research, 30(4), 718-748.

Mukhtar, U., Zhong, Z., Tian, B., Razzaq, A., \& Hina, T. (2018). Does rural-urban migration improve employment quality and household welfare? Evidence from 
Review of Economics and Development Studies, Vol. 7 (1) 2021, 77-90

Pakistan. Sustainability, 10(11), 4281.

Philips, A. W. (1958). The relation between unemployment and the rate of change of money wage rates in the United Kingdom 1861-1957. Economica, 25(100), 283-299.

Qudrat-Ullah, H. (2015). Modelling and simulation in service of energy policy. Energy Procedia, 75, 2819-2825.

Qureshi, S. K., Ghani, E., \& Kazi, S. (1989). Employment Generation in Rural Pakistan with a Special Focus on Rural Industrialization: A Preliminary Analysis [with Comments]. The Pakistan Development Review, 28(4), 587-602.

Reardon, T., Berdegué, J., \& Escobar, G. (2001). Rural nonfarm employment and incomes in Latin America: overview and policy implications. World development, 29(3), 395-409.

Reddy, A. B. (2016). Labour force participation of elderly in India: patterns and determinants. International Journal of Social Economics.

Sargent, T. J. (1978). Estimation of dynamic labor demand schedules under rational expectations. Journal of Political Economy, 86(6), 1009-1044.

Senadza, B. (2012). Non-farm income diversification in rural Ghana: Patterns and determinants. African Development Review, 24(3), 233-244.

Shah, N., \& Soomro, B. A. (2017). Investigating entrepreneurial intention among public sector university students of Pakistan. Education+ Training.

Shaheen, R., Shabir, G., Faridi, M. Z., \& Yasmin, F. (2015). Determinants of female employment status in Pakistan: A case of Sahiwal District. Pakistan Journal of Commerce and Social Sciences (PJCSS), 9(2), 418-437.

Sheikh, K. H., Iqbal, Z., \& Akhtar, M. R. (1992). Short-Term Employment Functions in Manufacturing Industries: An Empirical Analysis for Pakistan [with Comments]. The Pakistan Development Review, 31(4), 1267-1277.

Siddiqui, D. A., \& Ahmed, Q. M. (2013). The effect of institutions on economic growth: A global analysis based on GMM dynamic panel estimation. Structural Change and Economic Dynamics, 24, 18-33.

Solon, G. (1999). Intergenerational mobility in the labor market. In Handbook of labor economics (Vol. 3, pp. 1761-180o). Elsevier.

Su, H., Dreesmann, N. J., Hough, C. L., Bridges, E., \& Thompson, H. J. (2021). Factors associated with employment outcome after critical illness: Systematic review, meta-analysis, and meta-regression. Journal of Advanced Nursing, 77(2), 653-663.

Sun, R., \& Wang, W. (2017). Transformational leadership, employee turnover intention, and actual voluntary turnover in public organizations. Public Management Review, 19(8), 1124-1141.

Tansel, A., \& Acar, E. Ö. (2017). Labor mobility across the formal/informal divide in Turkey. Journal of Economic Studies.

Unay-Gailhard, İ., \& Bojnec, Š. (2019). The impact of green economy measures on rural employment: Green jobs in farms. Journal of Cleaner Production, 208, 541-551.

ur Rahman, S., Chaudhry, I. S., \& Farooq, F. (2018). Gender inequality in education and household poverty in Pakistan: A case of Multan district. Review of Economics and Development Studies, 4(1), 115-126.

Westerhuis, G. K., \& Henrekson, M. (2016). The institutional evolution of labour market institutions in Europe and entrepreneurship. Financial and Institutional Reforms for an Entrepreneurial Society (FIRES).

Xiao, J., Boschma, R., \& Andersson, M. (2018). Industrial diversification in Europe: The differentiated role of relatedness. Economic Geography, 94(5), 514-549.

Zellner, A. (1962). An efficient method of estimating seemingly unrelated regressions and tests for aggregation bias. Journal of the American statistical Association, 57(298), 348-368. 